\title{
Study of regenerative breaking control for HEV with multispeed transmission
}

\author{
Jeewook Huh ${ }^{1}$, Kyoungcheol Oh ${ }^{1}$, Deokkeun Shin ${ }^{1}$ \\ ${ }^{1}$ Hyndai Motor Company, Jangduk-dong, Hwasung-si, Gyeonggi-do 445-70
}

\begin{abstract}
This study describes method of regenerative breaking control for HEV with multispeed transmission. When a gear is shifted during regenerative breaking, almost all TMED HEVs in the world lose decelerate linearity or reduce regenerative breaking to get decelerate linearity. So fuel efficiency is get down. We made concept that is called regenerative breaking practical quantity. Regenerative breaking practical quantity solved problem of decelerate linearity. And this technology is used in all TMED HEVs of Hyundai motor group. Regenerative breaking practical quantity reflect a characteristic curves of motor, shifting process of multispeed transmission and following of hydraulic brake for cooperative control of HCU, MCU, TCU, AHB and so on. As a result of this technology, decelerate linearity is retained for sale the HEV. Furthermore fuel efficiency is improved about $8.4 \%$ because regenerative breaking quantity is increased about $28 \%$.
\end{abstract}

Keywords: HEV(Hybrid Electric Vehicle), TMED(Transmission Mounted Electric Device), Actual regenerative

breaking, Characteristic curves of motor, Decelerate linearity

\section{Introduction}

Regenerative braking system is an energy recovery system which converts kinetic energy of vehicle into electric energy to maximize fuel efficiency. The electric energy is changed by controlling counter torque of a motor, and the energy is stored in a high voltage battery to reuse for driving vehicle. Thus, the regenerative braking system is a core technology for high fuel efficiency of green cars with electric motors in them such as Hybrid Electric Vehicle (HEV), Electric Vehicle (EV), Fuel Cell Electric Vehicle (FCEV) and so on. Comparing to conventional vehicle, about $30 \%$ of the effect of improving HEV's fuel efficiency comes from the regenerative braking system. [1]

TMED (Transmission Mounted Electric Device) HEV system is decelerated by using regenerative braking from a motor located at transmission input shaft and hydraulic brake equipment installed on the wheels and referenced as in Figure 1.

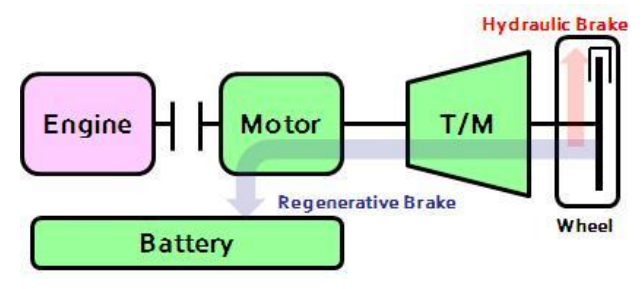

Figure 1: Braking system of TMED

This means that breaking feeling change is easily occurred during shifting gear due to different location of regenerative braking and hydraulic braking. Regenerative braking is located at input shaft of transmission, and hydraulic braking is located at output shaft of transmission. Therefore, it is necessary to develop technology of minimizing the brake feeling change during shifting gear while braking. Nowadays, every other company's HEVs applied TMED system has not only large brake feeling change during shifting gear while braking but also difficult control, so increase of fuel efficiency is limited from regenerative braking. 
To control the regenerative braking system, it is essential to use cooperative control with $\mathrm{HCU}$ (Hybrid Control Unit) that calculates the actual regenerative braking, AHB(Active Hydraulic Booster) that decides hydraulic braking, and TCU(Transmission Control Unit) that control shifting gear.[2]

The actual regenerative braking is defined as the braking torque on the wheels. It is very important to measure accurate amount of regenerative braking during gear shifting.

Also, conventional control logic applied the actual regenerative braking causes a problem that storage limit of battery and motor is occurred as a motor capacity is over $30 \mathrm{~kW}$. The problem leads to change the actual regenerative braking suddenly, so it is hard to make linear brake and increase fuel efficiency. Thus, using the conventional control logic is impossible for vehicle applied over $30 \mathrm{~kW}$ motor, and developing new control logic for the actual regenerative braking is needed for vehicle installed the large capacity of battery and motor to improve fuel efficiency.

In addition, TMED HEV only operates regenerative braking during shifting gear. It is difficult to calculate the actual regenerative braking, so new control logic for the actual regenerative braking considering shift characteristic is necessary to calculate the actual regenerative braking accurately.

Lastly, braking feeling is different at the same brake force since vehicle load is changed like climbing and descending situation. For that reason, logic is needed considering the vehicle load.

\section{Body}

\subsection{Cooperative control method for Regenerative braking system}

During regenerative braking, cooperative control is needed between controllers. HCU, AHB, and TCU are main, and MCU (Motor Control Unit) and BMS (Battery Management System) is additionally used and referenced as in Figure 2.[3]

When driver wants to decelerate and actives brake pedal (Pedal Simulator)[4], AHB decides total amount of brake according to demand brake. Next, AHB also decides permissible amount of regenerative braking from front brake after AHB distribute brake force to front and rear wheel.

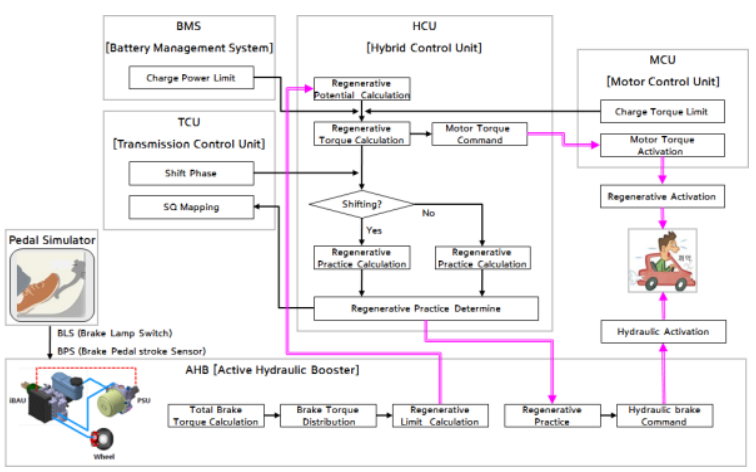

Figure 2: Regenerative braking flow

HCU get the information of battery and motor states. From the information, HCU determines the regenerative braking torque based on permissible amount of regenerative braking taken from AHB and sends it to MCU. Considering the shifting gear information from TCU, HCU decides the actual regenerative braking which is real regenerative braking torque applied to wheel and sends to AHB. At steady state, motor response is faster than hydraulic brake response, so HCU maintains linear brake thereby changing hydraulic brake following the motor torque and the actual regenerative braking easily.

MCU outputs regenerative braking torque to motor and actives regenerative braking.

AHB operates hydraulic braking as the actual regenerative braking is excluded from demand brake.

To minimize the actual regenerative braking change transmitted to output shaft during shifting gear, TCU minimizes transmission output torque change by using torque intervention, transmission clutch, and brake slip and transmits regenerative braking torque to wheel as the actual regenerative braking.

\subsection{Calculation of the actual regenerative braking}

The actual regenerative braking $\left(\right.$ Regen $\left._{\mathrm{AV}}\right)$ is amount of brake transmitted motor torque $\left(\mathrm{T}_{\text {motor }}\right)$ to wheel through transmission. If gear is not shifted, the actual regenerative braking can be represented as (1). GR is gear ratio.

However, due to not only changing gear ratio but also occurring slip inside the transmission brake and clutch during shifting gear, it is impossible to calculate the actual regenerative braking from (1). Therefore, control method for the actual regenerative braking during shifting gear is developed.

$$
\operatorname{Re} \text { gen }_{A V}=T_{\text {motor }} \times G R
$$




\subsection{Control the actual regenerative braking during shifting gear}

To make good brake feeling during regenerative brake with shifting gear, HCU leads hydraulic brake to follow the actual regenerative braking. Also, HCU transmits the actual regenerative braking by calculating real motor torque delivered to wheel to make linear break. Thus, the role of HCU is important to make good brake feeling. TCU have to transmit torque as much as the actual regenerative braking from torque intervention, brake slip, and clutch slip.

Since the actual regenerative braking is calculated during shifting gear, motor characteristic is considered. Motor characteristic can be divided to steady power state and steady torque state. Steady power state means that motor has constant power and steady torque state means that motor has constant torque. The criteria speed of separating two states is called base rpm and referenced as in Figure 3. [5]

As the actual regenerative braking is controlled during shifting gear, three modes can be classified.

First is steady power mode that motor is always operated at over base rpm during shifting gear.

Second is steady torque mode that motor is always operated at under base rpm during shifting gear.

The last one is mode change that motor is operated during shifting gear while motor changes steady power state to steady torque state or opposite situation.

Calculating the actual regenerative braking at each mode is as follows.

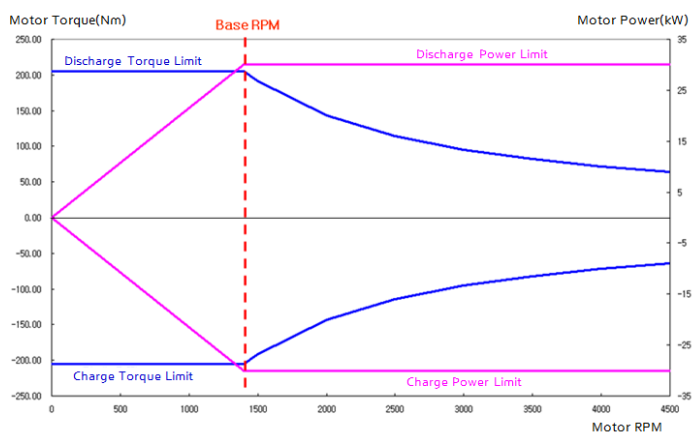

Figure 3: Motor characteristic curve

\subsubsection{Steady power mode}

Gear ratio at steady power mode $\left(\mathrm{GR}_{\text {cal_SteadyPower }}\right)$ is calculated from transmission input speed $\left(\omega_{\text {TmIn }}\right)$ and output speed $\left(\omega_{\text {TmOut }}\right)$ following (2), that is near value to real gear ratio. Because motor torque is changed following motor speed at steady power state, the actual regenerative braking during gear shifting can be consisted by using gear ratio calculated from motor torque and speed change, and it is possible to make good brake feeling and referenced as in Figure 3.

Motor torque used at calculating the actual regenerative braking $\left(\right.$ Regen $\left._{\text {SteadyPower }}\right)$ is motor torque before intervention from TCU, not real motor torque after torque intervention. Motor torque after torque intervention is used to control motor torque transmitted to wheel, so using motor torque after torque intervention is limited to find the actual regenerative braking. Since motor torque before torque intervention is operated as same motor characteristic curve, it is useful to calculate the amount of actual regenerative braking.

$G R_{\text {cal_SteadyPower }}=\frac{\omega_{\text {TmIn }}}{\omega_{\text {TmOut }}}$

Re gen $_{\text {SteadyPower }}=T_{\text {motor }} \times G R_{\text {cal }}$

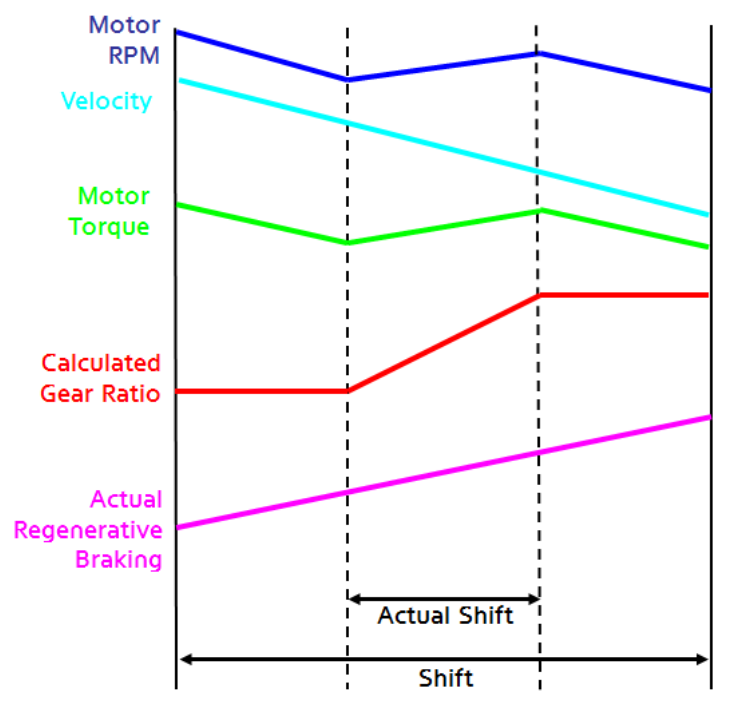

Figure 4: Actual regenerative braking at steady power mode

The result of the actual regenerative braking at steady power mode has same change range like Figure 4 during shifting gear, and hydraulic brake has constant change. As the result, stable decelerate is possible without uncomfortable brake feeling.

\subsubsection{Steady torque mode}

At steady torque mode, motor always output constant torque before and after shifting gear, so change amount of regenerative braking is calculated from gear ratio. However, if the actual regenerative braking is calculated from real gear ratio at steady power mode, hydraulic brake cannot 
follow and it is difficult to map shift quality due to sudden amount change of active regenerative braking. Thus, increasing gear ratio in constant gradient makes comfortable brake feeling after shifting gear starts. Gradient of gear ratio is considered shift step ratio and shifting time. In other words, the actual regenerative braking can be linear by keeping constant gear ratio gradient at shifting gear.

As motor torque before intervention is not followed changing motor speed at steady torque mode, gear ratio change affects to change actual regenerative braking at calculating the actual regenerative braking $\left(\right.$ Regen $\left._{\text {SteadyTorque }}\right)$. Gradient of gear ratio $(\alpha)$ is found from shifting time $\left(\Delta \mathrm{T}_{\text {Shift }}\right)$ and differential ratio $\left(\mathrm{GR}_{\text {Diff }}\right)$ which is difference between gear ratio before $\left(\mathrm{GR}_{\text {before}}\right)$ and after $\left(\mathrm{GR}_{\mathrm{after}}\right)$ shifting gear, and calculated gear ratio $\left(\mathrm{GR}_{\text {cal_SteadyTorque }}\right)$ according to gradient can be measured by integrating the gear ratio before shifting gear.

$$
\begin{aligned}
& G R_{\text {Diff }}=G R_{\text {after }}-G R_{\text {before }} \\
& \alpha=\frac{G R_{\text {Diff }}}{\Delta T_{\text {Shift }}}
\end{aligned}
$$

$G R_{\text {cal_SteadyTorque }}=G R_{\text {before }}+\int_{0}^{T_{\text {Shiffend }}} \alpha d T$

Re gen $_{\text {SteadyTorque }}=T_{\text {motor }} \times G R_{\text {cal_SteadyTorque }}$

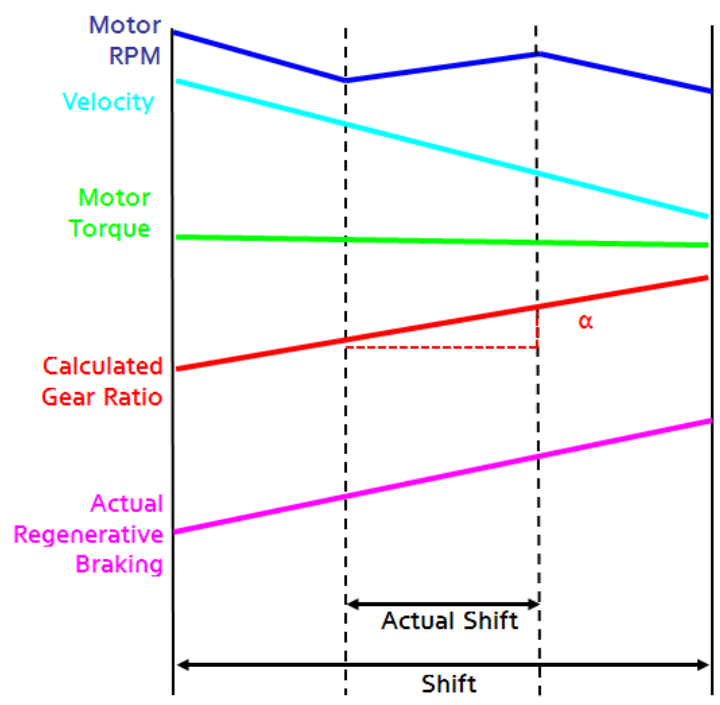

Figure 5: Actual regenerative braking at steady torque mode

\subsubsection{Mode change}

Mode change is case that motor speed passes the base rpm at shifting gear during regenerative braking. In other words, motor change steady power mode to steady torque state and change to steady power state again at real shifting gear.

If motor speed decreases at steady power state, motor torque also decrease, and then motor speed drops. Next, motor operates at steady torque state, and motor torque has constant value. This means that motor characteristic is changed while shifting gear, so it is necessary to control considering that state is steady power state or steady torque state.

In case of mode change, motor operates to enter steady power state at real shifting gear. However, since entering time is different because of shifting time, deceleration, starting regenerative braking, and motor torque and speed, it is difficult to map motor motion. In addition, frequent mode change causes making linear brake is hard. As the result, after entering steady power state, virtual motor torque $\left(\mathrm{T}_{\text {motor_virtual }}\right)$ is found from fixing motor torque at steady torque state and gear ratio is found by using incline gear ratio gradient. Virtual motor torque and gear ratio is used for controlling and referenced as in Figure 6.

Gear ratio is calculated considering steady power state and steady torque state. Gear ratio gradient at steady torque state is found by gradient $\alpha$ calculated at steady torque state and gradient $\beta$ calculated at steady power state. Gear ratio at calculating the actual regenerative braking is found from incline gear ratio gradient $\gamma$ and referenced as in Figure 7. Calculated gear ratio $\left(\mathrm{GR}_{\text {cal_ModeChange }}\right)$ is derived from gear ratio before shifting gear and ४. (8) Calculated gear ratio and virtual motor torque is used to calculate the actual regenerative braking at mode change (Regen ModeChange $_{\text {) }}$. (9)

By using virtual motor torque, the actual regenerative braking at mode change can be risen stability, and brake feeling is comfortable because of hydraulic brake following and referenced as in Figure 8.

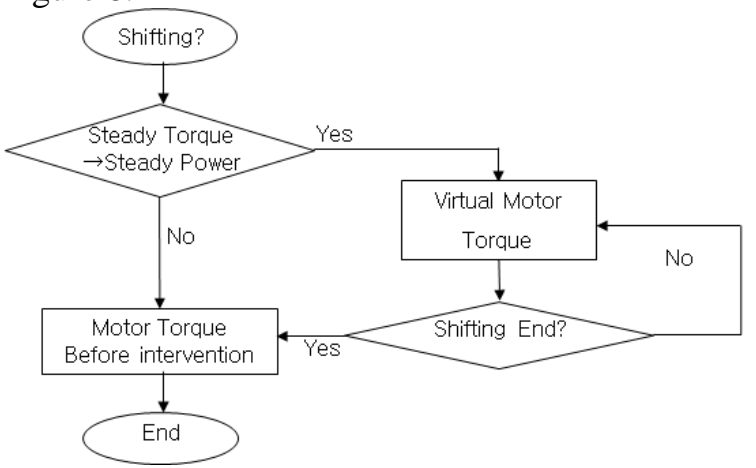

Figure 6: Motor torque flow chart at mode change 


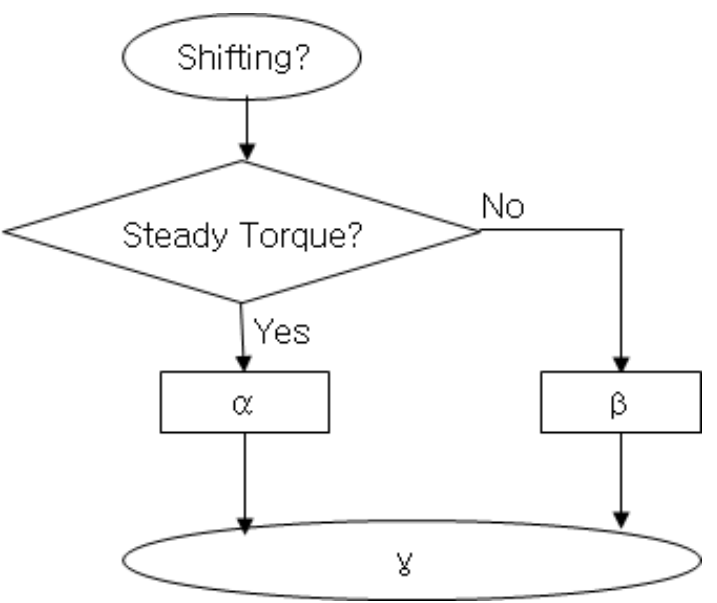

Figure 7: Gear ratio decision at mode change

$G R_{\text {cal_ModeChange }}=G R_{\text {before }}+\int_{0}^{T_{\text {ShifEnd }}} \gamma d T$
$\operatorname{Re}$ gen $_{\text {ModeChange }}=T_{\text {motor_virtual }} \times G R_{\text {cal_ModeChange }}$

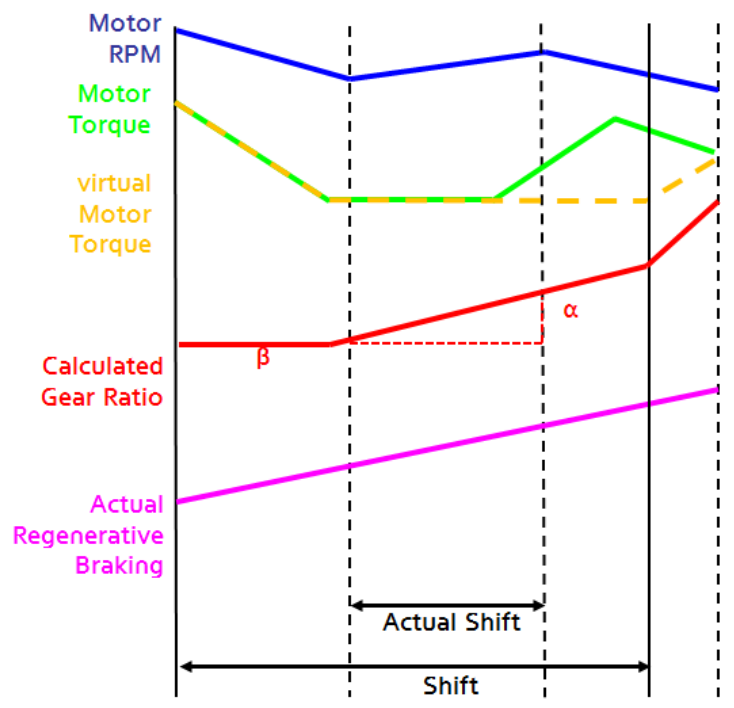

Figure 8: Actual regenerative braking at Mode change

\subsection{Logic development of the actual regenerating braking}

Motor and battery used at regenerative battery has charge/discharge limit due to environment. In case of battery, battery charge limit following the lowest or highest SOC (State of Charge), temperature, or trouble with battery. Motor also has limit because of temperature, motor speed, and motor trouble. For example, battery has charge limit before pre-heating in winter, so it is hard to charge by motor, and regenerative brake cannot work. Like this charge limit, motor is changed characteristic and this situation affects to regenerative braking.
Base rpm is motor speed that is criteria of motor characteristic change. Based on base rpm, low speed state is steady torque state not considering motor speed, and high speed state is steady power state also not considering motor speed. Motor torque has the characteristic of 2 nd order curve with motor speed. If motor torque increases, motor speed decreases.

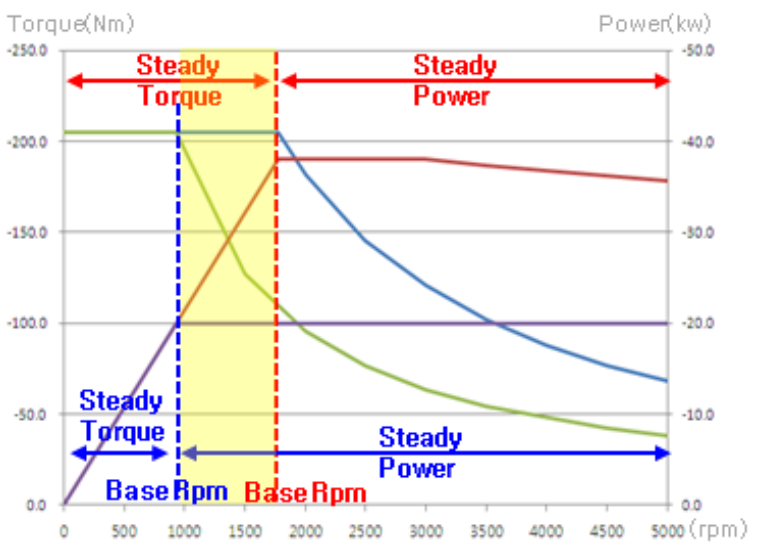

Figure 9: Motor characteristic curve when motor has limit or not

Figure 9 is about the motor characteristic curve when motor has limit or not. Steady power/torque characteristic is followed red at no limit situation, but steady power/torque characteristic is followed blue at limit situation. Thus, base rpm is shifted by charge limit, and yellow part is motor torque characteristic change section. Motor characteristic change leads to problem that it is hard to calculate the actual regenerative braking accurately due to wrong motor characteristic. Conventional control is not considered motor charge limit, so the actual regenerative braking is calculated over estimation due to basing on steady state. This causes uncomfortable brake feeling. To solve this problem, the actual regenerative braking control is developed to calculate accurate value, so linear brake is possible.

While the actual regenerative braking is calculated, charge limit can estimate from MCU transmitted allowable motor charge maximum power and BMS transmitted allowable battery charge maximum power $\left(\mathrm{P}_{\text {BatChgLmt }}\right)$.

Motor power limit for battery power $\left(\mathrm{P}_{\text {MotChgLmt2Bat }}\right)$ is calculated considering efficiency of motor/invertor/battery (Eff MotInvBat $)$ (10) As battery is charged by motor, charged power to battery decreased because of efficiency of motor/invertor/battery

$P_{\text {MotChgLme Bat }}=P_{\text {BatChgLmt }} \div$ Eff $f_{\text {MotInvBat }}$

As the actual regenerative braking is motor torque transferred to the wheel, rechargeable power 
should be converted to torque. Motor charging torque limit $\left(\mathrm{Tq}_{\mathrm{ChgLmtWh}}\right)$ is calculated using motor charging power limit $\left(\mathrm{P}_{\text {MotChgLmt }}\right)$ and wheel speed(transmission output shaft speed $\left(\mathrm{N}_{\mathrm{Whl}}\right)$.)(11) The reason for calculating by the wheel speed not the motor speed is that the transmission output shaft speed is steady while the transmission input shaft speed is variable during transmission. Furthermore control of input shaft speed is used to secure shift quality (SQ). Thus, problems such as rapid change of motor torque, the actual regenerative braking and rough shift quality are prevented despite rapid change of motor. In brief, the problem of the actual regenerative braking during the limited recharge state is solved by calculating the actual regenerative braking using wheel speed.

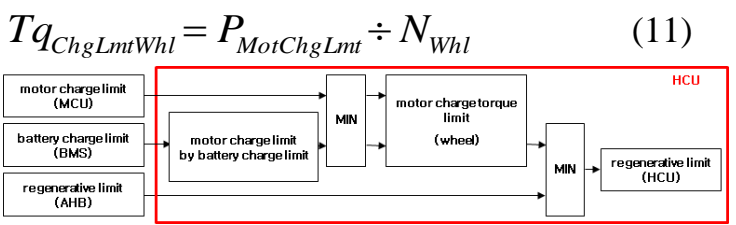

Figure 10: Regenerative braking limit control flow

Like Figure $10 \mathrm{HCU}$ calculate motor charging torque limit $\left(\mathrm{Tq}_{\mathrm{ChgLmtWhl}}\right)$ by calculating with criteria of wheel rechargeable power of motor and battery with criteria of wheel from MCU and BMS. And HCU finally compute the available amount of regenerative braking considering the allowable amount of regenerative braking from EBS.

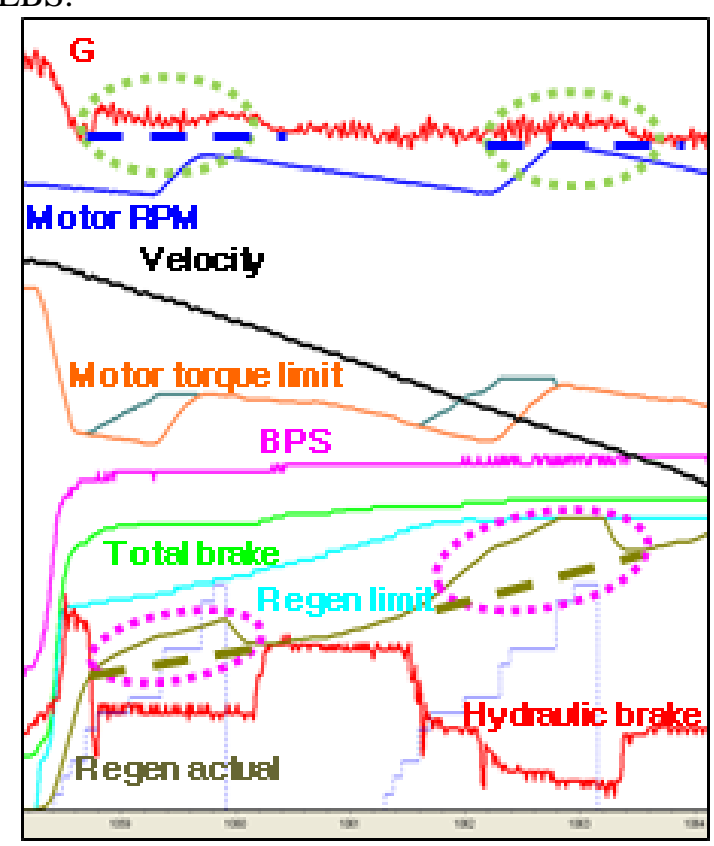

Figure 11:before/after by battery charge limit logic
Figure 11 shows that the actual regenerative braking is excessively calculated than the actual amount of regenerative braking transferred to the wheel because motor characteristic curve was not reflected previously during transmission and misjudged constant power region to constant torque region when battery recharge limit was made. Thus braking delay is caused by reduction of deceleration due to excessively decreased hydraulic brake.

However, the stable amount of regenerative braking can be calculated like dotted line when logic of the available amount of regenerative braking is applied because motor characteristic curve is reflected and it judge constant power region. Therefore rapid change of hydraulic brake is prevented and braking linearity is secured like dotted line because it minimizes the fluctuation of deceleration.

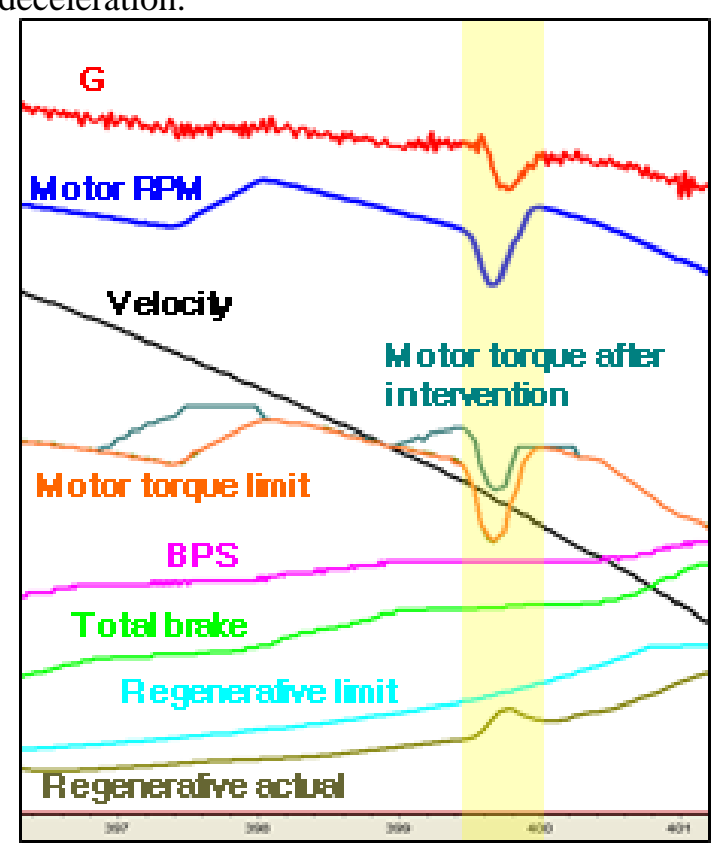

Figure 12: before/after by TM input speed logic

Figure 12 is a comparison of before and after the available amount of regenerative braking logic application when input shaft speed is rapidly changed during transmission. Deceleration changes rapidly because the transmission loosening phenomenon makes rapid change of input shaft speed and that makes motor torque and the actual regenerative braking change rapidly in previous logic. Also rapid change of motor torque worsens the transmission loosening phenomenon and that makes not only variation of deceleration due to rapid change of the actual regenerative braking but also decreased shift quality. When logic of the available amount of regenerative braking is applied, motor torque command is 
maintained as a dotted line and the amount of regenerative braking is increased constantly even if the transmission loosening phenomenon is caused. Therefore, braking linearity and shift quality can be secured.

\subsection{Regenerative braking mode decision considering storage limit}

The regenerative braking mode is used for actual regenerative braking where regenerative braking mode is classified to constant power region, constant torque region and mode shift by motor characteristic map.

Constant power mode is operated when motor speed is over base RPM, constant torque mode is operated when motor speed is under base RPM and mode shift is operated when motor speed is on base RPM. It is essential to make a decision the regenerative braking mode, because the way to calculate actual regenerative braking is different depending on the motor mode.

When limiting the charging battery, base RPM of motor is able to be changed. It has an advantage to assure linear actual regenerative braking. Hence constant torque and power region is correctly separated by calculating the base RPM $\left(\mathrm{N}_{\text {Base }}\right)$ (12), from motor charging power limit $\left(\mathrm{P}_{\text {MotChgLmt }}\right)$ and motor maximum charging torque $\left(\mathrm{Tq}_{\mathrm{Max}}\right)$.

$$
N_{\text {Base }}=P_{\text {MotChgLmt }} \div T q_{\text {Max }}
$$

The decision of regenerative braking mode is comprised of constant power mode, constant torque mode and mode shift. The gear ratio should be obtained in constant torque and mode shift at beginning of transmission shifting. Hence, the regenerative braking mode should be determined considering motor speed, transmission shifting time and deceleration, once the transmission shifts and referenced as in Figure 13.

Constant power mode is defined when the motor speed is in constant power region, which is over base RPM $\left(\mathrm{N}_{\text {Base }}\right)$. Constant power mode is decided when Minimum motor speed $\left(\mathrm{N}_{\text {motor }}\right)$ is over base RPM on shifting. Motor speed is a minimum when transmission begins to shift during braking. Hence, the decrement of motor speed $\left(\Delta \mathrm{N}_{\text {SteadyPower }}\right)$ is calculated from demanded braking torque and transmission state. If the motor speed, when a transmission begins to shift, is larger than the sum of base RPM and the decrement of motor speed, the regenerative braking mode is decided as the constant power mode.
For instance, Regenerative braking mode is constant power mode when base RPM is 1400 RPM, decrement of motor is $200 \mathrm{RPM}$ and motor speed is over $1600 \mathrm{rpm}(13)$.

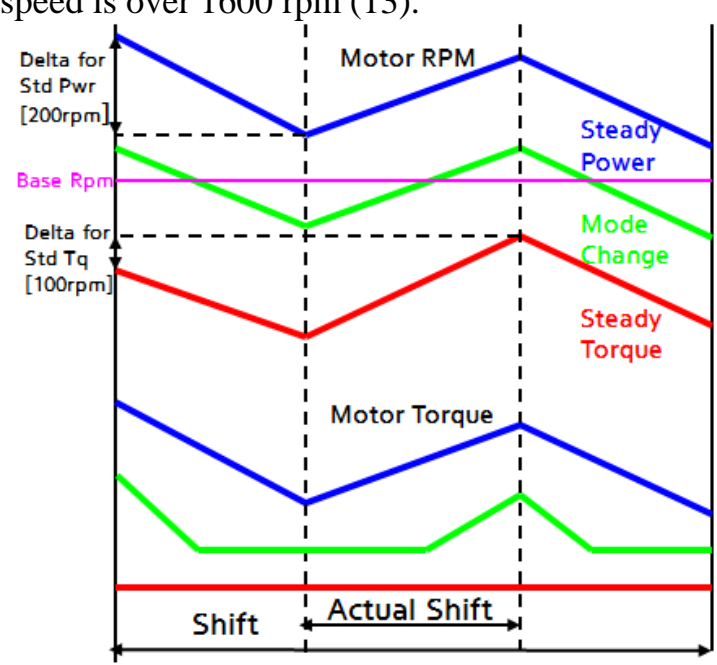

Figure 13: Regenerative braking mode decision

$N_{\text {Motor }}>N_{\text {base }}+\Delta N_{\text {SteadyPowe }}$

Constant torque mode is defined when the motor speed is in constant torque region, which is under base RPM $\left(\mathrm{N}_{\text {Base }}\right)$. Constant torque mode is decided when Maximum motor speed $\left(\mathrm{N}_{\text {motor }}\right)$ is under base RPM on shifting. Motor speed is a maximum when transmission finishes shifting during braking. Hence, the change amount of motor speed $\left(\Delta \mathrm{N}_{\text {SteadyTorque }}\right)$ is calculated from demanded torque and transmission state. If the motor speed is smaller than sum of base RPM and the change amount of motor speed, the regenerative braking mode is decided as the constant torque mode.

For instance, Regenerative braking mode is constant torque mode when base RPM is 1400 RPM, change amount of motor is -100 RPM and motor speed is under 1300 RPM (14)

$N_{\text {Motor }}<N_{\text {base }}+\Delta N_{\text {SteadyTorque }}$

Mode change is defined when the motor speed is cross the base RPM ( $\mathrm{N}_{\text {Base }}$ ).(15)

$$
\begin{aligned}
& N_{\text {base }}+\Delta N_{\text {ModeChangkow }}<N_{\text {Motor }} \\
& <N_{\text {base }}+\Delta N_{\text {ModeChangHigh }}
\end{aligned}
$$

\subsection{Regenerative braking mode decision considering gear ratio}

Logic development of regenerative braking considering the gear ratio maintains linear brake. Calculating the amount of regenerative braking and control is classified to 'steady power mode, steady torque mode and mode change'. Different 
motor speed between before speed change and after speed change decide each mode.

But interval between gear ratio is different from gear number to number, so amount of speed changing is also different and referenced as in Figure 14. To decide the regenerative braking mode need an algorithm of high accuracy mode decision from using motor speed with considering the gear ratio.

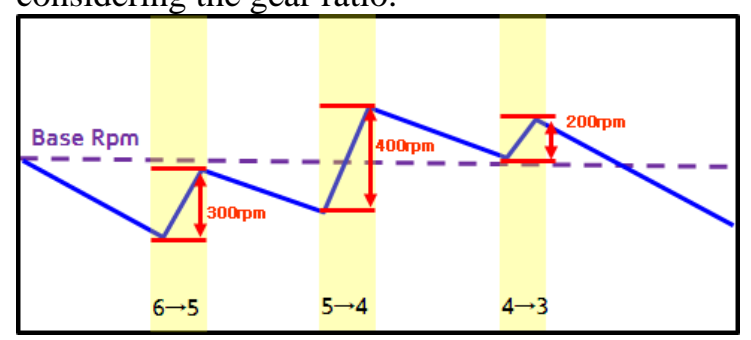

Figure 14: Motor speed by TM gear

Table 1: Method for decision of shifting mode

\begin{tabular}{|c|c|c|c|}
\hline 5 & 6 & 5 & 4 \\
\hline 4 & $6-5$ & & \\
\hline Target & & $5-4$ & \\
\hline 3 & & $5-3$ & $4-3$ \\
\hline
\end{tabular}

Regenerative braking has 6-5/5-4/5-3/4-3 gear shift before stop. Controller should consider a shifting mode and speed changing at each time. Gear shift mode is decided by target gear number and current gear number. The method of each gear shift mode decision is shown in the table 1 below.

\subsection{Regenerative braking mode decision considering vehicle load}

The load of the vehicle is changed depending on the number of people and luggage. In this case, although the drivers apply the same brake position, deceleration of the vehicle is different. Since reduction of the vehicle change while shifting gears, when the vehicle load changes, the case of misjudgement occurs when applying the mode set based on the normal vehicle load. That is, since the motor speed as a reference for determining the each regenerative braking mode varies according to the vehicle load, the logic considering this point is needed. Therefore, by determining the regenerative braking mode via the vehicle load determined by the dual mode by calculating the vehicle load during shifting gear while braking, a more accurate determination mode is possible.

If the load increase, because of the deceleration is large compared to the normal load conditions, the possibility that the logic enters the mode change increases, even if the transmission is initiated from the same motor speed in steady power region and referenced as in Figure 15.

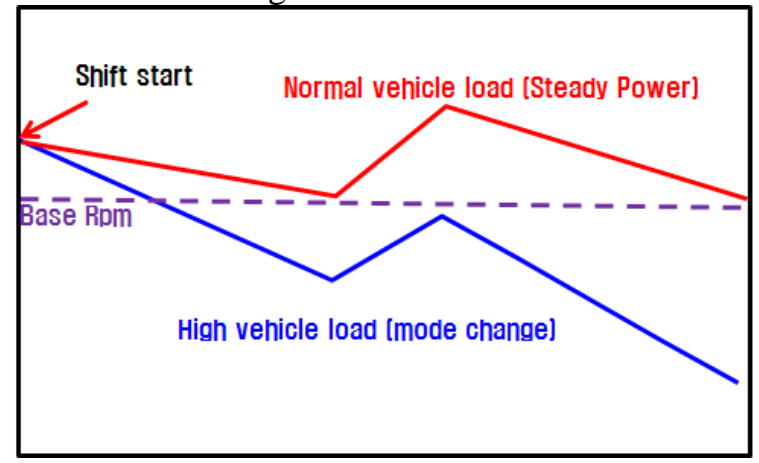

Figure 15: motor speed in steady power region

In addition, in case the load may be small, since deceleration is less than that in the normal load condition, the possibility that if enters the mode change increases, even If they start shifting from the same motor speed steady torque region and referenced as in Figure 16.

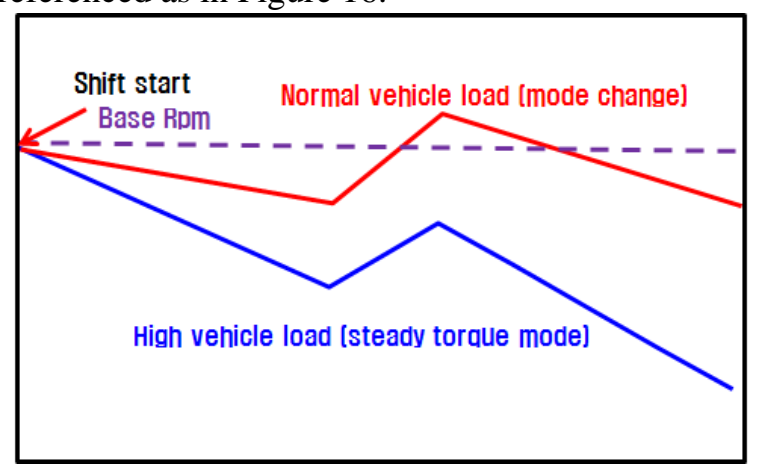

Figure 16: motor speed in steady torque region

That is, even if the shifting gear begins at the same motor speed, it is possible to secure a more improved control by determining the regenerative braking mode as vehicle load.

Therefore, we divide the load to nine steps. In the case of high load, we determined the mode by adding the $\alpha$-factor in variation of motor speed from the starting moment of shifting gear to the end. (16), (17)

In the case of low load, we determined the mode by adding the $\beta$-factor in variation of motor speed from the starting moment of shifting gear to the end. (18), (19) 
$N_{\text {Motor }}>N_{\text {base }}+\left(\Delta N_{\text {SteadyPowe }}+\alpha\right)$

$N_{\text {base }}+\left(\Delta N_{\text {ModeChangbow }}+\alpha\right)<N_{\text {Motor }}$

$<N_{\text {base }}+\left(\Delta N_{\text {ModeChangHigh }}+\alpha\right)$

$N_{\text {base }}+\left(\Delta N_{\text {ModeChangkow }}+\beta\right)<N_{\text {Motor }}$

$<N_{\text {base }}+\left(\Delta N_{\text {ModeChangtigh }}+\beta\right)$

$N_{\text {Motor }}>N_{\text {base }}+\left(\Delta N_{\text {SteadyTorque }}+\beta\right)$

\section{Conciusion}

This paper aim to construct a more robust logic in order to improve fuel economy and get the linearity of braking for TMED type HEV equipped with auto transmission. It can calculate linearly the actual regenerative braking with regard to various conditions and gear ratios

(1) The control of active regenerative braking gets more fuel economy than competitor's without increasing production cost and a driver's safety to decrease fluctuation of deceleration feeling. Its control is our core technology, which is expected to use original technology continuously for our eco-friendly cars to be produced equipped with discrete gears transmission. Moreover, its regenerative braking is improved about $28 \%$ and its fuel economy $8.4 \%$ based on Federal Test Procedure (FTP)

(2) The development on the logic of active regenerative braking improves factor of deterioration of fuel economy which cannot estimate the amount of regenerative braking and deteriorate linear braking; a sudden change of regenerative braking affects the hydraulic braking in the case that maximum torque of regenerative braking varies as a limit of battery and motor to restrict the power. Specifically, on $7{ }^{\circ} \mathrm{C}$ cold FTP, there is amount $0.5 \%$ improvement of fuel economy. Drivability becomes 7 from $6+$ on cold and 7+ from 7 on basic condition. Shift quality and braking feeling can be improved because of not generating the fluctuation of motor toque and active regenerative braking using the actual regenerative braking calculated by velocity of output shaft as the control of releasing input shaft for shifting quality in regenerative braking.

(3) Accurate decision of regenerative braking mode can be able to get linearly the actual regenerative braking applying the control logic of mode decision considering motor and limit of charging battery.

Fluctuation of shifting quality is solved and development period is reduced if the mode is determined by ratio of each gear as regenerative braking mode decision considering shifting gear ratio. Drivability improves $7+$ from 7 .

(4) Linearity of braking is proposed by improving the actual regenerative braking for vehicle load; driving downhill or uphill, emergency stopping, taking a lot of passengers or loading cargo. Fuel economy especially improves to be able to regenerative brake on driving uphill

\section{References}

[1] Hanbyeol Jin, Development of Regenerative Braking Control Logic for a HEV Performance Simulator, A Fall Conference of KSAE 2007, pp. 2087-2092, 2007

[2] Eunha Lee, Regenerative braking co-operative control system for eco-friendly vehicle, A Fall Conference of KSAE 2013, pp. 458-459, 2013

[3] 3. Junzhi Zhang, Cooperative control of regenerative braking and hydraulic braking of an electrified passenger car, Journal of Automobile Engineering 2012, pp. 1289-1302, 2012

[4] 4. Hoon Yeo, Development of Stroke Simulator for a Hybrid Electric Vehicle A Spring Conference of KSAE 2001, pp. 760-765, 2001

[5] 5. Sanghoon Kim, DC,AC,BLDC Motor Control, BOGDOO, pp. 394-404, 2nd

\section{Authors}

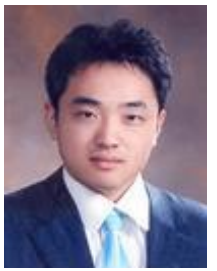

Jeewook Huh

Research Engineer

Hybrid Electric vehicle Performance

Development Team

Research \& Development Division,

Hyundai Motor Group

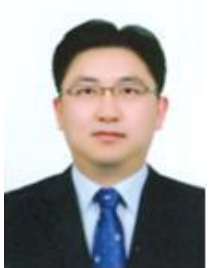

Kyoungcheol Oh

Senior Research Engineer

Hybrid Electric vehicle Performance

Development Team

Research \& Development Division,

Hyundai Motor Group

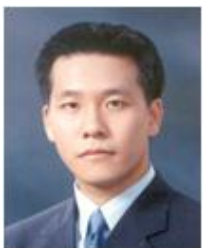

Deokkeun Shin

Senior Research Engineer

Electric vehicle Performance

Development Team

Research \& Development Division,

Hyundai Motor Group 